\title{
From intermediate anisotropic to isotropic friction at large strain rates to account for viscosity thickening in polymer solutions
}

Journal Article

Author(s):

Stephanou, Pavlos S.; Kröger, Martin (1)

Publication date:

2018-05-14

Permanent link:

https://doi.org/10.3929/ethz-b-000267579

Rights / license:

Creative Commons Attribution-NonCommercial 4.0 International

Originally published in:

The Journal of Chemical Physics 148(18), https://doi.org/10.1063/1.5019337 


\section{From intermediate anisotropic to isotropic friction at large strain rates to account for viscosity thickening in polymer solutions}

Cite as: J. Chem. Phys. 148, 184903 (2018); https://doi.org/10.1063/1.5019337

Submitted: 13 December 2017 . Accepted: 23 April 2018 . Published Online: 11 May 2018

Pavlos S. Stephanou (D), and Martin Kröger (D)
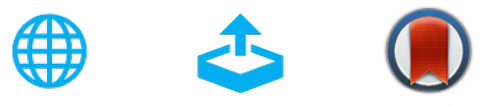

View Online

Export Citation

\section{ARTICLES YOU MAY BE INTERESTED IN}

\section{Single polymer dynamics for molecular rheology}

Journal of Rheology 62, 371 (2018); https://doi.org/10.1122/1.5013246

Communication: A coil-stretch transition in planar elongational flow of an entangled polymeric melt

The Journal of Chemical Physics 148, 141103 (2018); https://doi.org/10.1063/1.5026792

\section{Composition inversion in mixtures of binary colloids and polymer}

The Journal of Chemical Physics 148, 184902 (2018); https://doi.org/10.1063/1.5023393

Where in the world is AIP Publishing? Find out where we are exhibiting next 


\title{
From intermediate anisotropic to isotropic friction at large strain rates to account for viscosity thickening in polymer solutions
}

\author{
Pavlos S. Stephanou ${ }^{1}$ and Martin Kröger ${ }^{2}$ \\ ${ }^{1}$ Department of Mathematics and Statistics, University of Cyprus, P.O. Box 20537, 1678 Nicosia, Cyprus \\ ${ }^{2}$ Department of Materials, Polymer Physics, ETH Zürich, CH-8093 Zürich, Switzerland
}

(Received 13 December 2017; accepted 23 April 2018; published online 11 May 2018)

\begin{abstract}
The steady-state extensional viscosity of dense polymeric liquids in elongational flows is known to be peculiar in the sense that for entangled polymer melts it monotonically decreases-whereas for concentrated polymer solutions it increases-with increasing strain rate beyond the inverse Rouse time. To shed light on this issue, we solve the kinetic theory model for concentrated polymer solutions and entangled melts proposed by Curtiss and Bird, also known as the tumbling-snake model, supplemented by a variable link tension coefficient that we relate to the uniaxial nematic order parameter of the polymer. As a result, the friction tensor is increasingly becoming isotropic at large strain rates as the polymer concentration decreases, and the model is seen to capture the experimentally observed behavior. Additional refinements may supplement the present model to capture very strong flows. We furthermore derive analytic expressions for small rates and the linear viscoelastic behavior. This work builds upon our earlier work on the use of the tumbling-snake model under shear and demonstrates its capacity to improve our microscopic understanding of the rheology of entangled polymer melts and concentrated polymer solutions. Published by AIP Publishing. https://doi.org/10.1063/1.5019337
\end{abstract}

\section{INTRODUCTION}

Since the introduction of the tube/reptation model by de Gennes, Doi, and Edwards, ${ }^{1-3}$ this mean-field theory has established itself as a most capable tool in an attempt to interpret the dynamical nonequilibrium behavior of entangled (high molecular weight) polymer melts and concentrated polymer solutions. The incorporation of additional mechanisms that were originally omitted, like contour length fluctuations accounting for the dynamical evolution of the primitive path contour length, and constraint release (CR) accounting for the dynamical release of entanglements, $, 2,4$ allowed for the precise description of linear viscoelastic (LVE) properties. ${ }^{4-7}$ However, and despite the persevering efforts of polymer scientists to improve upon it in the non-linear regime, by incorporating, e.g., chain stretch due to elongation of the tube by the flow, ${ }^{8}$ finite extensibility, ${ }^{9}, 10$ and convective constraint release (CCR) accounting for a flow-induced release of entanglements, ${ }^{10-13}$ it still lacks consistency with available rheological data. In spite of the overall accepted effectiveness, and partial success, of the tube/reptation model, it is not the sole formalism that aims addressing the molecular origins of high molecular weight polymeric systems. Curtiss and Bird proposed a model for entangled polymer melts and concentrated polymer solutions, the tumbling-snake model,${ }^{14}$ derived via the use of the Curtiss-Bird-Hassager phase-space formulation for the kinetic theory of undiluted polymers, ${ }^{15}$ and contrary to Doi and Edwards (DE), it does not invoke a mean-field tube. The tumbling-snake model bears more similarities with sliplink models ${ }^{16,17}$ than with tube models, since slip link chains tumble. The tumbling-snake model entails, like the original tube/reptation one, the solution of a Fokker-Planck (FP) for the single-link distribution function, $f(\sigma, \mathbf{u}, t)$, which describes the probability that at time $t$ a chain segment at contour position $\sigma$ $\in[0,1]$ along the chain is oriented in direction $\mathbf{u}$, with $\mathbf{u}$ and $\sigma$ independent dynamical variables, and $\mathbf{u} \cdot \mathbf{u}=1$. The model employs the so-called independent alignment assumption (IAA), according to which chain segments are independently oriented by the imposed flow field. Reptation is identified as a one-dimensional diffusion process along the polymer's backbone, counteracting orientational diffusion corresponding to $\mathrm{CR}$ events, whose strength is parameterized by $\varepsilon^{\prime}$. The extra stress tensor contains a term due to the anisotropy of the friction tensor $\boldsymbol{\zeta}=\zeta_{\mathrm{eq}}[\boldsymbol{\delta}-(1-\boldsymbol{\varepsilon}) \mathbf{u u}]$ involving the link tension coefficient $\varepsilon \in[0,1]$; if $\varepsilon=0$, there is no friction against motion in the direction $\mathbf{u}$, whereas for $\varepsilon=1$, the friction tensor is isotropic as for an individual sphere. Despite the different assumptions made by the two formalisms, the original tube/reptation model is obtained as a special case $\varepsilon^{\prime}=\varepsilon=0$ of the more general FP equation of the tumbling-snake model ${ }^{15,18,19}$ (see also Ref. 20). Curtiss-Bird and their co-workers had worked out the analytically tractable non-tumbling model (when $\left.\varepsilon^{\prime}=0\right) .{ }^{15,18,19,21,22}$ Up to now, an analytic solution to the complete Curtiss-Bird model (with $\varepsilon^{\prime}>0$ ) is not known.

Recently, we provided a simple Brownian Dynamics (BD) solution scheme for the tumbling-snake model for $\varepsilon^{\prime}>0$ and applied it to both steady-state ${ }^{20}$ and time-dependent shear flow ${ }^{14,23}$ and for steady-state and time-dependent planar elongation. ${ }^{24}$ This solution scheme entails the numerical integration of two coupled Itô stochastic differential equations for the variables $\mathbf{U}_{t}$ (segment unit vector at time $t$ ) and $\sigma_{t}$ $\in[0,1]$ (relative contour position at time $t$ ) [see Eq. (5) of Ref. 24]. Given that in the absence of flow both $\mathbf{U}_{t}$ and $\sigma_{t}$ are 
uniform random variables, the tumbling-snake model is in accordance with the fluctuation-dissipation theorem..$^{25}$ These investigations provided ample evidence that the tumblingsnake model is able to capture the damping behavior of the transient viscosity in start-up shear experiments at high rates. ${ }^{26-28}$ At the same time, it predicts an absence of equally pronounced undershoots in both normal stress coefficients, again in accord with experimental data. ${ }^{14,23}$ The underlying reason for the appearance of this damping behavior was demonstrated to be caused by the shear-induced rotational motion of chains, ${ }^{23,28}$ further supported by non-equilibrium atomistic simulations. ${ }^{29-31}$ For this reason, similar undershoots are not expected to occur in elongational flows that are free from rotational contributions to the velocity gradient tensor. ${ }^{24} \mathrm{We}$ are going to calculate elongational flow properties using the parameters we employed earlier in a quantitative comparison with shear rheological data. To this end, the variable link tension coefficient is given as $\varepsilon=\varepsilon_{0} S_{2}^{2}$, 14,23 where $S_{2}$ denotes the 2 nd rank uniaxial nematic order parameter of polymer segments. ${ }^{32}$ It is worth recalling that the original Curtiss-Bird model with constant link tension coefficient violates the stress-optic rule (SOR), which is expected to hold close to equilibrium, and for that only small values for $\varepsilon$ should be employed. ${ }^{33}$ This problem has been eliminated in our work by using a variable link tension coefficient that automatically vanishes at equilibrium. Of course, at larger extensional rates, chain stretch becomes nonlinear and a failure of the SOR is measured. ${ }^{34}$

Prior to the BD formulation, other numerical approaches have been implemented to solve the Curtiss-Bird or tumblingsnake model with constant $\varepsilon$. One of us ${ }^{35}$ employed Galerkin's method to numerically solve the full, stationary tumblingsnake model subjected to steady shear flow, where trial functions were composed of spherical harmonics and Euler polynomials up to some finite order. A few more studies have been undertaken ${ }^{36-38}$ in which Currie's approximation ${ }^{39}$ of the Curtiss-Bird model is solved using a Galerkin's method. The use of a BD solution scheme is conceptually much simpler than these methods; it does not invoke a decoupling approximation and should therefore be clearly preferred over the earlier methods.

Experiments on entangled polysterene melts ${ }^{34,40}$ reveal that the extensional viscosity of polymer melts is monotonically decreasing with strain rate. On the other hand, the extensional viscosity of polymer solutions is seen to decrease below the inverse Rouse time and increase above. ${ }^{28,41-43}$ Such behavior was not predicted by the tube model even if the above-mentioned mechanisms, chain stretch, etc., were included. ${ }^{40,42,44}$

To remedy this shortcoming, a configuration-dependent monomeric friction coefficient had been introduced, which reflects the alignment of polymer chains. ${ }^{45-48}$ Its physical relevance may stem from nematic interactions between flow-aligned polymer chains and their surrounding solvent molecules. Aligned and strongly elongated chains experience reduced friction when they are placed parallel to each other, relative to the randomly oriented state under quiescent conditions. To account for this, the monomeric friction coefficient had been related to the uniaxial order parameter of the solution as follows: $\zeta=\zeta_{\text {eq }}$ when $0 \leq S \leq S_{c}$, whereas $\zeta=\zeta_{\text {eq }}\left(S / S_{c}\right)^{-1.25}$ when $S \geq S_{c}$; i.e., the monomeric friction coefficient is reduced only as soon as the order parameter exceeds a critical value $\left(S_{c} \approx 0.14\right) .{ }^{48}$ Usually, the order parameter is given as $S=[\phi+(1-\phi) \bar{\varepsilon}] S_{2}$, where $\bar{\varepsilon}$ is the nematic interaction parameter that originates from the assumption that the order parameter of solvent molecules, $S_{s}$, is proportional to the one of polymer segments, $S_{s}=\bar{\varepsilon} S_{2}$. This modification leads to a decrease in the extensional viscosity when $S \geq S_{c}{ }^{48}$ Such a modification has proven able of addressing the peculiar extensional behavior of polymer melts and solutions. ${ }^{28,41-43}$

The underlying idea was not completely new. Giesekus ${ }^{49}$ was the first to propose a coupling between friction and polymer conformation in polymer melts, followed by Bird and DeAguiar. ${ }^{50}$ In their work, it is not the friction coefficient that is reduced, but it is the friction tensor that becomes anisotropic. The same idea is reminiscent of the expression employed by Curtiss and Bird in the tumbling-snake model mentioned above. Invoking such a concept while deriving a rheological constitutive model for entangled polymer melts in the context of non-equilibrium thermodynamics, has shown promising. ${ }^{10}$

Thus, in this work, we revisit the contradiction between the rheological data for the extensional viscosity of polymer melts solutions and show that the link tension coefficient, given as $\varepsilon=\varepsilon_{0} S_{2}^{2}$, is able to predict this behavior in a qualitative manner, while the coefficient $\varepsilon_{0}$ decreases as the polymer concentration increases. The structure of this manuscript is as follows: In Sec. II, we provide the series expansion of the uniaxial elongation (UE) viscosity for the tumbling-snake model in the case of both steady-state and start-up UE for small, dimensionless elongation rates. In Sec. III, we solve the model numerically using BD simulations and further compare its predictions with available experimental data. We conclude with Sec. IV, where we discuss the significance of this work.

\section{SMALL ELONGATION RATE EXPANSION}

The time-dependent (extra or polymeric) stress tensor $\tau$ of the tumbling-snake model for a monodisperse polymer with polymerization degree $N$, temperature $T$, polymer number density $n$, subjected to a homogeneous flow field characterized by the transposed velocity gradient tensor $\boldsymbol{\kappa}$, is given by ${ }^{15,20}$

$$
\begin{aligned}
\frac{\tau(t)}{G}= & -\left(1-\varepsilon^{\prime}\right)\left(\langle\mathbf{u u}\rangle^{(1)}(t)-\frac{1}{3} \delta\right) \\
& -3 \varepsilon_{0}^{\prime}\left(\langle\mathbf{u u}\rangle^{(2)}(t)-\frac{1}{18} \delta\right) \\
& -\varepsilon \mathbf{B}(t),
\end{aligned}
$$

with modulus $G=n k_{\mathrm{B}} T(N-1)$ and $\varepsilon_{0}^{\prime}=\varepsilon^{\prime}(N-1)^{2}$, involving the following orientational averages calculated with the solution of the corresponding FP equation ${ }^{14}$ for the single-link orientational distribution function $f(\sigma, \mathbf{u}, t)$ 


$$
\begin{aligned}
\langle\mathbf{u u}\rangle^{(1)}(t) & =\int_{0}^{1} d \sigma \int d \mathbf{u} f(\sigma, \mathbf{u}, t) \mathbf{u u} \\
\langle\mathbf{u u}\rangle^{(2)}(t) & =\int_{0}^{1} \sigma(1-\sigma) d \sigma \int d \mathbf{u} f(\sigma, \mathbf{u}, t) \mathbf{u u}, \\
\mathbf{B}(t) & =\lambda \boldsymbol{\kappa}: \int_{0}^{1} \sigma(1-\sigma) d \sigma \int d \mathbf{u} f(\sigma, \mathbf{u}, t) \mathbf{u u u u},
\end{aligned}
$$

where $\int d \mathbf{u}$ denotes an integral over the unit sphere and $\lambda$ is a time constant proportional to $\zeta_{\mathrm{eq}} / k_{\mathrm{B}} T$, squared bond length $a$, and $N^{3+\beta}$, where $\beta$ is the chain constraint exponent. Note that the polymerization degree is related to the number of entanglements per polymer, $Z$, employed by DE via the relation $N=3 Z$. The so-called reptation of disengagement time is $\tau_{d}=\lambda / \pi^{2}$. For the case of uniaxial elongational flow at rate $\dot{\epsilon}$ in direction of unit vector $\mathbf{e}_{z}$, one has $\boldsymbol{\kappa}=\frac{1}{2} \dot{\epsilon}\left(3 \mathbf{e}_{z} \mathbf{e}_{z}-\delta\right)$.

\section{A. Steady-state uniaxial elongation}

We begin by considering a steady-state spherical harmonics expansion of the single-link distribution function around equilibrium under steady-state UE, assuming a constant $\varepsilon$. The methodology employed is described in the supplementary material (Sec. A), and the final expression for the expansion, up to 2nd order in the dimensionless Weissenberg number $\mathrm{Wi}=\dot{\epsilon} \lambda$, is given in Eq. (A6) of the supplementary material. The regime of strong flows $\dot{\epsilon} \tau_{R} \gg 1$ with Rouse time $\tau_{R}$ corresponds to $\mathrm{Wi} \gg N$. Upon inserting this expansion into the stress tensor Eq. (1), we obtain the UE viscosity, $\eta_{\mathrm{E}}=\left(\tau_{z z}-\tau_{x x}\right) / \dot{\epsilon}$, up to second order in Wi:

$$
\begin{aligned}
\frac{\eta_{\mathrm{E}}}{3 G \lambda}= & \frac{1}{60}\left(1+\frac{2}{3} \varepsilon\right)+\frac{4}{105}\left(\frac{3}{4}+\varepsilon\right) \Delta_{1} \mathrm{Wi} \\
& +\frac{4}{245}\left[\left(\frac{3}{4}+\varepsilon\right) \Delta_{2}-6(1-\varepsilon) \Delta_{3}\right] \mathrm{Wi}^{2},
\end{aligned}
$$

or alternatively, if we normalize with the zero-rate value,

$$
\begin{aligned}
\frac{\eta_{\mathrm{E}}}{3 \eta_{0}}= & 1+\frac{16}{7\left(1+\frac{2}{3} \varepsilon\right)}\left(\frac{3}{4}+\varepsilon\right) \Delta_{1} \mathrm{Wi} \\
& +\frac{48}{49\left(1+\frac{2}{3} \varepsilon\right)}\left[\left(\frac{3}{4}+\varepsilon\right) \Delta_{2}-6(1-\varepsilon) \Delta_{3}\right] \mathrm{Wi}^{2}
\end{aligned}
$$

Note that the zero-elongation UE viscosity follows Trouton law, according to which the zero-elongation UE viscosity is three times the zero-shear-rate viscosity, $\eta_{0}$. The following abbreviations have been introduced for numerical prefactors appearing in (3) and (4):

$$
\Delta_{j} \equiv 24 \sum_{v=1, \text { odd }}^{\infty} \frac{1}{(\pi v)^{4} k_{j}(v)} \quad(j=1,2,3),
$$

with the kernels $k_{1}(v)=K_{2}, k_{2}(v)=K_{2}^{2}$, and $k_{3}(v)=K_{2} K_{4}$ that depend on both $\varepsilon^{\prime}$ and $\varepsilon_{0}^{\prime}$ via

$$
K_{j} \equiv\left(1-\varepsilon^{\prime}\right)(\pi v)^{2}+j(j+1) \varepsilon_{0}^{\prime} .
$$

It is instructive to verify Eq. (4) for two limiting cases. In the first limit, $\varepsilon^{\prime}=0$, implying $K_{j}(v)=(\pi v)^{2}$, the $\Delta_{j}$ are readily evaluated, $\Delta_{1}=1 / 40$ and $\Delta_{2}=\Delta_{3}=17 / 6720$, and results should reduce to the tumbling-snake model with $\varepsilon^{\prime}=0$. We obtain

$$
\begin{aligned}
\frac{\eta_{\mathrm{E}}}{3 G \lambda}= & \frac{1}{60}\left(1+\frac{2}{3} \varepsilon\right)+\frac{1}{1050}\left(\frac{3}{4}+\varepsilon\right) \mathrm{Wi} \\
& +\frac{17}{58800}\left(\varepsilon-\frac{3}{4}\right) \mathrm{Wi}^{2},
\end{aligned}
$$

which is indeed identical to Eq. (19.6-16) in Ref. 15 (considering a monodisperse system) and Eq. (29) in Ref. 22. The expression (7) further reduces to the DE result ${ }^{1}$ for $\varepsilon=0$. In the second limit, $\varepsilon^{\prime}=1$ with $N=2$, the chain reduces to a rigid dumbbell. For this case, $K_{j}(v)=j(j+1)$ and all kernels $k_{j}$ are independent of $v$, leading to $\Delta_{1}=1 / 24, \Delta_{2}=1 / 144$, and $\Delta_{3}=1 / 480$. We thus obtain from Eq. (3)

$$
\begin{aligned}
\frac{\eta_{\mathrm{E}}}{3 G_{\mathrm{rd}} \lambda_{\mathrm{rd}}}= & \frac{3}{5}\left(1+\frac{2}{3} \varepsilon\right)+\frac{12}{35}\left(\frac{3}{4}+\varepsilon\right) \mathrm{Wi}_{\mathrm{rd}} \\
& +\frac{72}{175}\left(\varepsilon-\frac{3}{8}\right) \mathrm{Wi}_{\mathrm{rd}}^{2}
\end{aligned}
$$

with $\mathrm{Wi}_{\mathrm{rd}} \equiv \dot{\epsilon} \lambda_{\text {rd }}$, or alternatively,

$$
\begin{aligned}
\frac{\eta_{\mathrm{E}}}{3 \eta_{0, \mathrm{rd}}}= & 1+\frac{4}{7\left(1+\frac{2}{3} \varepsilon\right)}\left(\frac{3}{4}+\varepsilon\right) \mathrm{Wi}_{\mathrm{rd}} \\
& +\frac{24}{35\left(1+\frac{2}{3} \varepsilon\right)}\left(\varepsilon-\frac{3}{8}\right) \mathrm{Wi}_{\mathrm{rd}}^{2},
\end{aligned}
$$

where $\eta_{0 \text {,rd }}$ is the corresponding zero-rate viscosity for the rigid rod given by [see Eq. (11) of Ref. 20 for $\mathrm{Wi}=0$ ]

$$
\frac{\eta_{0, \mathrm{rd}}}{G_{\mathrm{rd}} \lambda_{\mathrm{rd}}}=\frac{3}{5}\left(1+\frac{2}{3} \varepsilon\right) \text {. }
$$

Here, as in Ref. 20, $G=6 G_{\text {rd }}$ and $\lambda=6 \lambda_{\text {rd }}$. For $\varepsilon=1$, Eq. (8) is also identical to Eq. (16.5) by Bird et al. ${ }^{51}$ for a rigid dumbbell. Our result, Eq. (8), generalizes the expression by Bird et $a l .{ }^{51}$ when hydrodynamic interaction is taken into account by identifying $\varepsilon=\lambda_{2}^{(2)} / \lambda_{2}^{(1)} \cdot 20$

If, instead of a constant link tension coefficient, we consider a variable link tension coefficient, given as $\varepsilon=\varepsilon_{0} S_{2}^{2},{ }^{14,23}$ then, up to second-order terms, we obtain

$$
\varepsilon=\varepsilon_{0} \frac{4}{25}\left(\Gamma_{1} \mathrm{Wi}\right)^{2},
$$

where $\Gamma_{1}$ is defined in Supplementary Eq. (A1b) of Ref. 23. Then, the UE viscosity is given as

$$
\begin{aligned}
\frac{\eta_{\mathrm{E}}}{3 G \lambda}= & \frac{1}{60}+\frac{\Delta_{1}}{35} \mathrm{Wi}+\frac{4}{245}\left(\frac{3}{4} \Delta_{2}-6 \Delta_{3}\right) \mathrm{Wi}^{2} \\
& +\frac{2 \varepsilon_{0}}{1125}\left(\Gamma_{1} \mathrm{Wi}\right)^{2}
\end{aligned}
$$

\section{B. Transient uniaxial elongation}

Next, we consider a time-dependent spherical harmonics expansion of the single-link distribution function around equilibrium to be able to obtain the LVE analytical predictions for constant $\varepsilon$; the procedure is described in the supplementary material (Sec. A); the final expression for the expansion of the time-dependent single-link distribution function, up to first order in Wi, is given by Eq. (A4) of the supplementary material. Inserting Eq. (A4) of the supplementary material into 
the stress tensor expression (1), we obtain analytical expressions for the time-dependent UE viscosity, $\eta_{\mathrm{E}}^{+}(t)$, which turns out to be three times the time-dependent shear viscosity (first presented in Ref. 14)

$$
\frac{\eta_{\mathrm{E}}^{+}(t)}{3 G \lambda}=\frac{1}{60}\left(1+\frac{2}{3} \varepsilon\right)-\frac{1}{15} \Delta_{0}(t),
$$

where the following abbreviation has been introduced:

$$
\Delta_{0}(t)=24 \sum_{\nu=1, \text { odd }}^{\infty} \frac{\exp \left(-K_{2} t / \lambda\right)}{(\pi v)^{4}} .
$$

Taking the rigid dumbbell limit, $\varepsilon^{\prime}=1$ and $N=2$, Eq. (13) becomes

$$
\frac{\eta_{\mathrm{E}}^{+}(t)}{3 G_{\mathrm{rd}} \lambda_{\mathrm{rd}}}=\frac{3}{5}\left(1+\frac{2}{3} \varepsilon\right)-\frac{3}{5} \exp \left(-t / \lambda_{\mathrm{rd}}\right)
$$

and reduces further to Eq. (18.9) of Ref. 51 for $\varepsilon=1$. Our Eq. (15) thus generalizes the expression by Bird et al. ${ }^{51}$ when hydrodynamic interaction is taken into account by identifying $\varepsilon$ as in Sec. II A.

If, instead of a constant link tension coefficient, we consider a variable link tension coefficient, given as $\varepsilon=\varepsilon_{0} S_{2}^{2}$, the least order expansion gives

$$
\varepsilon=\varepsilon_{0} \frac{4}{25} \mathrm{Wi}^{2}\left[\Gamma_{1}-\Gamma_{1}(t)\right]^{2},
$$

where $\Gamma_{1}(t)$ with $\Gamma_{1}(0)=\Gamma_{1}$ is defined in supplementary Eq. (A3b) of Ref. 23, which at small times simplifies to

$$
\varepsilon=\varepsilon_{0} \frac{9}{25}(\dot{\epsilon} t)^{2}
$$

As the variable link tension coefficient vanishes in the linear regime, the time-dependent UE viscosity turns out to be

$$
\frac{\eta_{\mathrm{E}}^{+}(t)}{3 G \lambda}=\frac{1}{60}-\frac{1}{15} \Delta_{0}(t) .
$$

Accordingly, at small times, the UE viscosity varies linearly with time,

$$
\frac{\eta_{\mathrm{E}}^{+}(t)}{3 G \lambda}=\frac{\varepsilon_{0}^{\prime}+2\left(1-\varepsilon^{\prime}\right)}{10} \frac{t}{\lambda},
$$

where we have made use of Eq. (B8) of the supplementary material of Ref. 14.

\section{COMPLETE SOLUTION USING BROWNIAN DYNAMICS SIMULATIONS}

The BD algorithm that we employ in this work is identical, apart from the different choice of flow field, $\boldsymbol{\kappa}$, with the one we had described in detail for both steady-state ${ }^{20}$ and transient shear flows. ${ }^{14,23}$

\section{A. Steady-state uniaxial elongation}

The steady-state link tension coefficient as a function of dimensionless elongation rate Wi for $N=100(Z \approx 33)$ and various values of $\varepsilon_{0}^{\prime}$ is shown in Fig. 1. At small elongation rates, $\varepsilon$ is seen to increase quadratically with $\mathrm{Wi}$, in accord with Eq. (11). On the other hand, at large shear rates, $\varepsilon \rightarrow \varepsilon_{0}$, irrespective of the value of $\varepsilon_{0}^{\prime}$. This is expected since for UE flow $S_{2}=\left\langle u_{z}^{2}-u_{x}^{2}\right\rangle^{(1)}$, and as $\mathrm{Wi} \rightarrow \infty$, then $\left\langle u_{z}^{2}-u_{x}^{2}\right\rangle^{(1)} \rightarrow 1$. The model predictions when $\varepsilon=\varepsilon_{0} S_{2}^{2}$ at large elongation rates

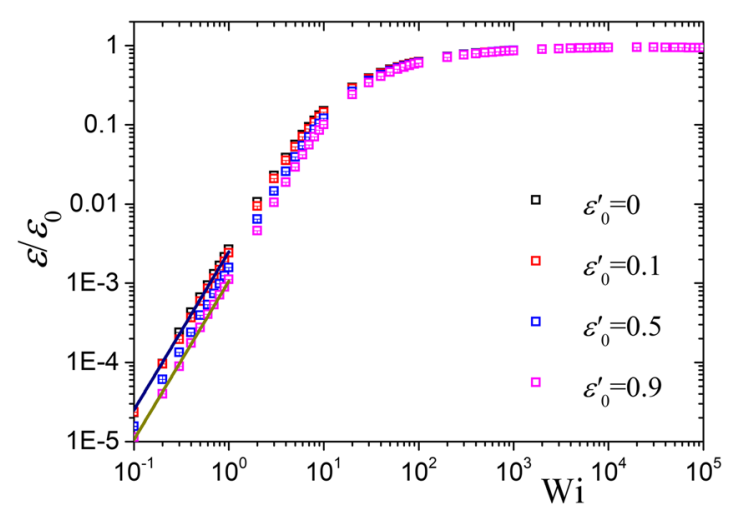

FIG. 1. Predictions for the link tension coefficient, $\varepsilon / \varepsilon_{0}$, as a function of dimensionless elongation rate Wi for $N=100(Z \approx 33$ entanglements) and various values of $\varepsilon_{0}^{\prime}$. The thick lines give the predictions of Eq. (11) when $\varepsilon_{0}^{\prime}=0$ (dark blue) and 0.9 (dark yellow).

are thus identical with the ones for a constant link tension coefficient.

The reduced steady-state UE viscosity as a function of the dimensionless elongation rate is presented in Fig. 2. Panel (a) of this figure shows the variation of UE viscosity upon changing $\varepsilon_{0}^{\prime}$ while keeping $N=100(Z \approx 33)$ and $\varepsilon=0$ fixed. We first note that, as dictated by Eq. (12), the UE viscosity at small elongation rates is independent of the value of $\varepsilon_{0}^{\prime}$ and follows Trouton's law. However, the UE viscosity increases with increasing $\varepsilon_{0}^{\prime}$ after about $\mathrm{Wi} \approx 3$ and seems to be passing from a maximum when $\varepsilon_{0}^{\prime}>0$ (see inset). The power-law part of the curves is unaffected by the value of $\varepsilon_{0}^{\prime}$ and is always equal to -1 , as for the DE model. ${ }^{1}$ In Fig. 2(b), we show the same variation as in Fig. 2(a) but now with $\varepsilon_{0}=0.1$. Again, the UE viscosity at small elongation rates follows Eq. (12) and a maximum is seen when $\varepsilon_{0}^{\prime}>0$. All curves after the maximum reach monotonically, irrespective of the value of $\varepsilon_{0}^{\prime}$, the same value of the UE viscosity at large elongation rates. This value, as first discussed by Bird et al., ${ }^{22}$ is simply equal to

$$
\frac{\eta_{\mathrm{E}}(\infty)}{3 G \lambda}=\frac{\varepsilon_{0}}{18} \Rightarrow \frac{\eta_{\mathrm{E}}(\infty)}{3 \eta_{0}}=\frac{10 \varepsilon_{0}}{3},
$$

which for $\varepsilon_{0}=0.1$ gives $\eta_{\mathrm{E}}(\infty) /(3 G \lambda)=1 / 180$. Equation $(20)$ can be easily derived by noting that as $\mathrm{Wi} \rightarrow \infty$, then

$$
\begin{aligned}
\left\langle u_{z}^{2}-u_{x}^{2}\right\rangle^{(1)} & \rightarrow 1, \\
\left\langle u_{z}^{2}-u_{x}^{2}\right\rangle^{(2)} & \rightarrow 1 / 6, \\
B_{z z}-B_{x x} & \rightarrow \mathrm{Wi} / 6 .
\end{aligned}
$$

When $\varepsilon_{0}>0$, the third term in the stress tensor expression, Eq. (1), dominates at large elongation rates, leading to the leveling-off of the UE viscosity at a value given by Eq. (20). Note that both Eqs. (20) and (21) apply also when $\varepsilon_{0}^{\prime}>0$. Further increasing the value of the parameter $\varepsilon_{0}$, for given $\varepsilon_{0}^{\prime}$ and $N$, keeps the small elongation rate predictions almost unaffected [Figs. 2(c) and 2(d)]; as noted from Eq. (12), only second-order terms in $\mathrm{Wi}$ are to be affected by the value of $\varepsilon_{0}$. At large $\mathrm{Wi}$, the curves reach the value of the reduced $\eta_{\mathrm{E}}(\infty)$, Eq. (20). When the value of $\varepsilon$ exceeds $3 / 10$, then $\eta_{\mathrm{E}}(\infty)>3 \eta_{0}$ [Fig. 2(d)]. Finally, it should be stressed that the theoretical expressions given by Eq. (12) not only provide the zero-shearrate asymptotes but also the downturn as Wi is further increased 

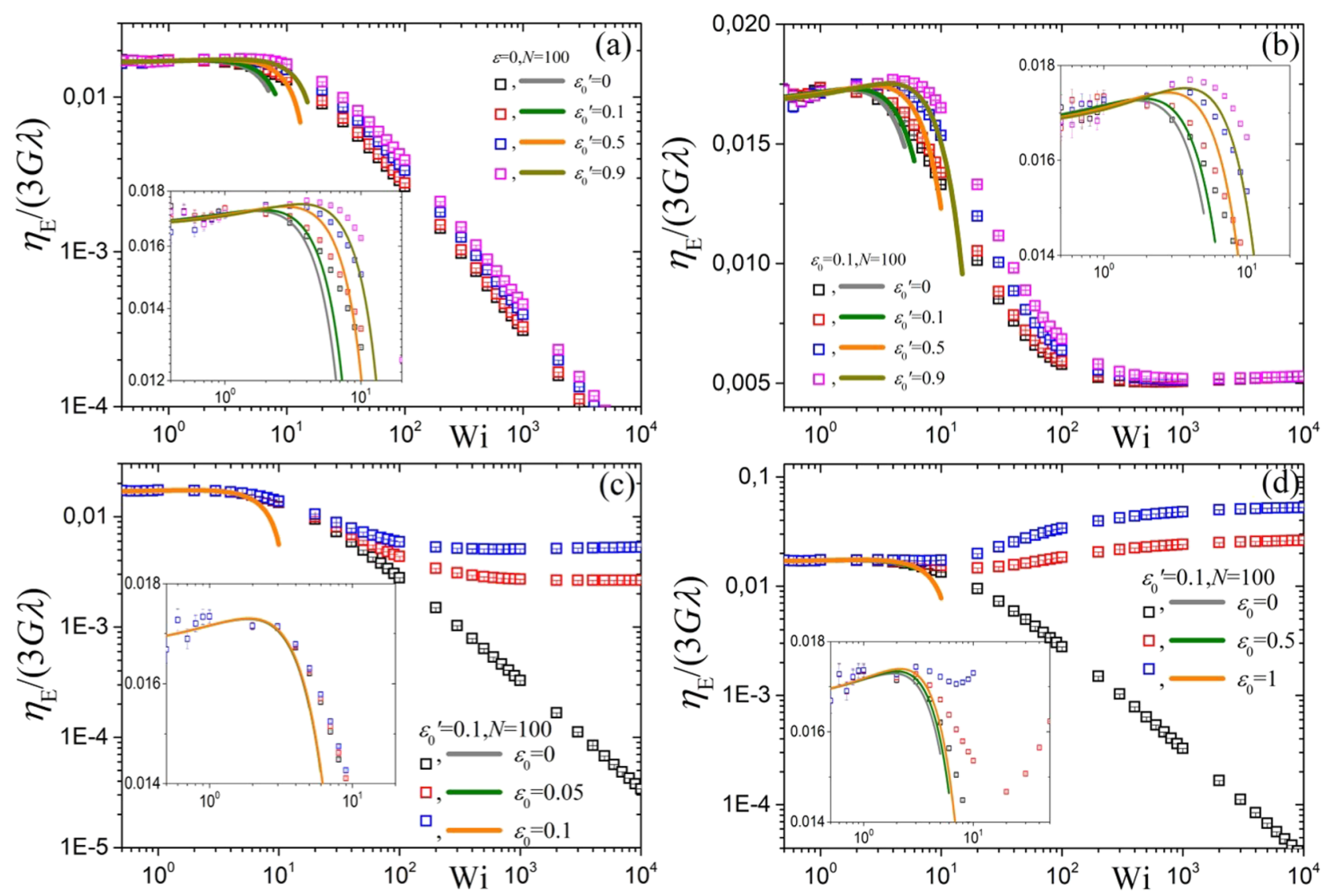

FIG. 2. Predictions for $\eta_{\mathrm{E}} /(3 G \lambda)$ as a function of dimensionless elongation rate Wi for $N=100$ and various values of the parameters $\varepsilon_{0}$ and $\varepsilon_{0}^{\prime}$. The thick lines give the predictions of Eq. (12).

(see insets in all parts of Fig. 2). The corresponding predictions when the link tension coefficient is considered constant are given in Fig. (S1) of the supplementary material.

This feature is important as it predicts the behavior of polymer solutions, according to which at very high dilutions, the extensional viscosity increases, and at intermediate concentrations, it seems to be reaching a plateau after the inverse Rouse time ${ }^{28,41-43}$ (see also Fig. 5). We thus elaborate on it further. As Wi $\rightarrow \infty$, then $\left\langle u_{z}^{2}\right\rangle^{(1)} \rightarrow 1,\left\langle u_{x}^{2}\right\rangle^{(1)} \rightarrow 0$, and $\varepsilon \rightarrow \varepsilon_{0}$, implying $\zeta_{z z}=\varepsilon_{0} \zeta_{\text {eq }}$ and $\zeta_{x x}=\zeta_{\text {eq }}$; for the DE model (i.e., $\varepsilon=0$ ), the friction at the stretching direction is always zero, whereas in the case of the tumbling-snake model it is not; note that $\zeta_{z z} \approx \zeta_{x x}$ in the limit $\varepsilon_{0} \rightarrow 1$. The increase in the friction in the direction of stretching, controlled by the parameter $\varepsilon_{0}$, leads to an increase in the extensional viscosity needed to capture the experimental data (see Sec. III C). We recall that within the presented theory the friction tensor for dilute polymer solutions is isotropic only at equilibrium and at large strain rates, while its maximum anisotropy is achieved at intermediate Wi because $\varepsilon=\varepsilon_{0} S_{2}^{2}$ changes more quickly than $\langle\mathbf{u u}\rangle^{(1)}$.

\section{B. Transient uniaxial elongation}

Next, we inspect the transient link tension coefficient, $\varepsilon / \varepsilon_{0}$, as a function of dimensionless time $t / \lambda$ for $N=100$ and various values of the parameter $\varepsilon_{0}^{\prime}$ and dimensionless elongation rates Wi (Fig. 3). At early times, this coefficient follows $\frac{9}{25}(\dot{\epsilon} t)^{2}$, irrespective of Wi and $\varepsilon_{0}^{\prime}$, whereas at larger times it monotonically approaches the steady-state values.

In Fig. 4, we show the transient UE viscosity as a function of the dimensionless time for various dimensionless elongation rates along with the LVE prediction, Eq. (18). For all elongation rates, we notice that as $t \rightarrow 0$ the UE viscosity $\eta_{\mathrm{E}}^{+} /(3 G \lambda)$ varies linearly with time, as prescribed by Eq. (19); i.e., it shifts towards smaller times with increasing $\varepsilon_{0}^{\prime}$. By using a link tension coefficient given as $\varepsilon=\varepsilon_{0} S_{2}^{2}$, we have thus amended the problematic predictions of the original

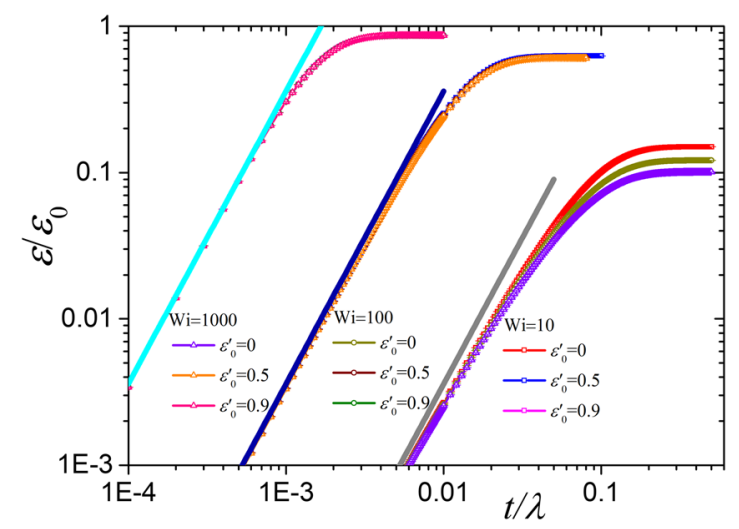

FIG. 3. Predictions for the link tension coefficient, $\varepsilon / \varepsilon_{0}$, as a function of dimensionless time for $N=100$ and various values of the parameter $\varepsilon_{0}^{\prime}$ and dimensionless elongation rate Wi. The thick straight lines give the predictions of $\frac{9}{25}(\dot{\epsilon} t)^{2}$. 

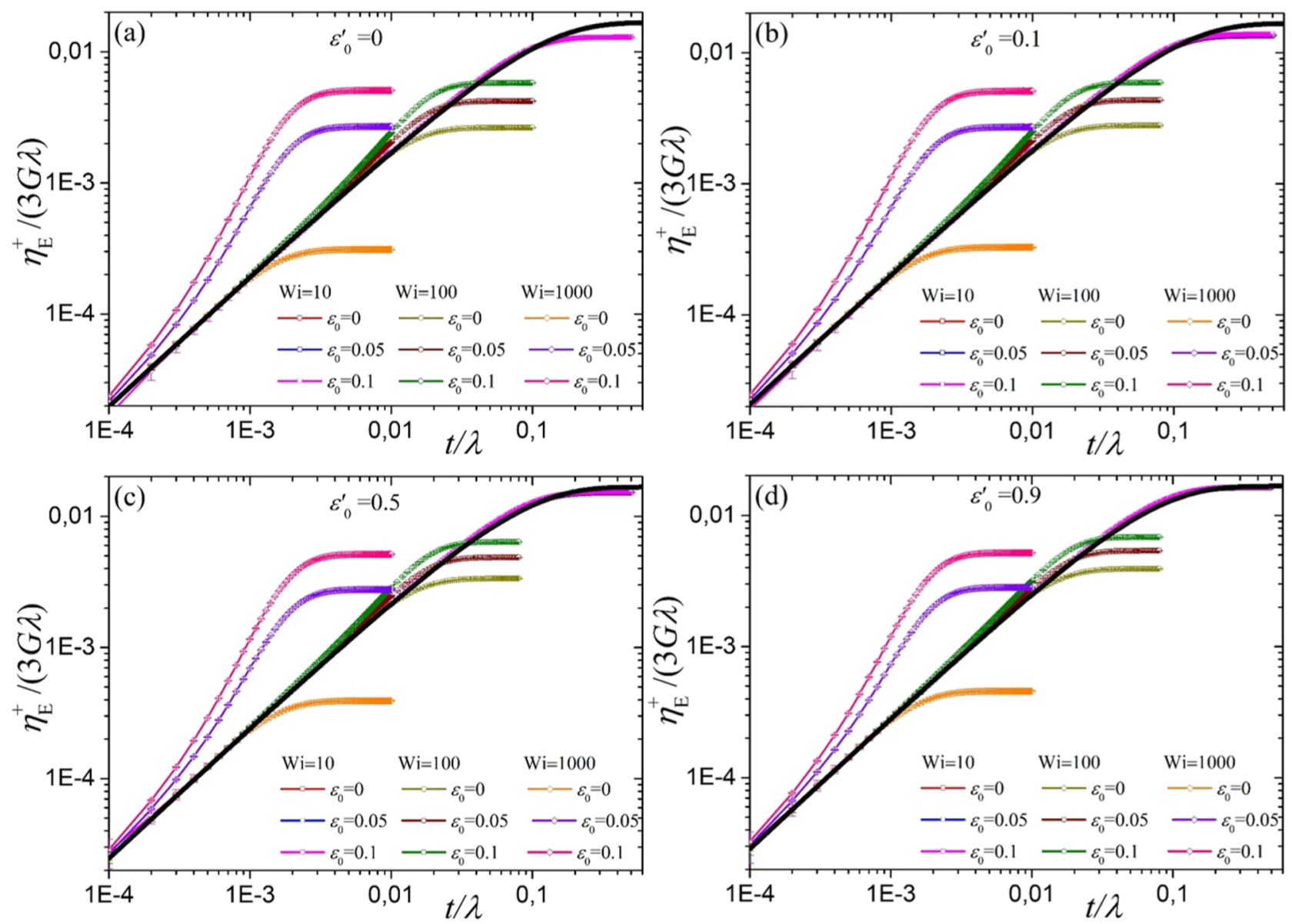

FIG. 4. Predictions for $\eta_{\mathrm{E}}^{+}(t) /(3 G \lambda)$ as a function of time for $N=100$ and various values of the parameter $\varepsilon_{0}$ and dimensionless elongation rate Wi for (a) $\varepsilon_{0}^{\prime}=0$, (b) $\varepsilon_{0}^{\prime}=0.1$, (c) $\varepsilon_{0}^{\prime}=0.5$, and (d) $\varepsilon_{0}^{\prime}=0.9$. The thick lines give the predictions of Eq. (18).

tumbling-snake model, employing a constant link tension coefficient, according to which $\eta_{\mathrm{E}}^{+} /(3 G \lambda)$ approaches the constant value $\varepsilon / 30$, irrespective of the value of the parameter $\varepsilon_{0}^{\prime}$ (see Sec. B of the supplementary material). As we further increase the elongation rate $(\mathrm{Wi}=100)$, the UE viscosity goes over the LVE prediction only when $\varepsilon_{0}>0$ but still reaches the steadystate value without reaching an overshoot, independently of the value of the parameters $\varepsilon_{0}^{\prime}>0$ and $\varepsilon_{0}$. This behavior can be contrasted with the predictions of the tumbling-snake model in simple shear in which a clear undershoot is observed for all values of the parameters $\varepsilon_{0}^{\prime}>0$ and $\varepsilon_{0}$ (cf. Fig. 1 in Ref. 14 and Fig. 6 in Ref. 23). The same trend is seen at a larger elongation rate $(\mathrm{Wi}=1000)$, but now the curves depart much sooner and more intensely from the LVE prediction. It is worthwhile mentioning that identical parameter values are used in the present Fig. 4 and in Fig. 6 of Ref. 23.

Overall, the major observation is that at large times all viscosity curves reach monotonically their steady-state values without presenting an undershoot, irrespective of the value of $\varepsilon_{0}^{\prime}$ and Wi. This is noteworthy since $\varepsilon_{0}^{\prime}>0$ in shear flow produces undershoots at large shear rates due to the tumbling behavior of polymer chains imposed by the rotational contribution to $\kappa$. The absence of a rotational contribution in UE hinders the appearance of undershoots in the UE viscosity, in concert with experimental data. The tumbling-snake model is seen to accurately capture this behavior without further readjustment even when $\varepsilon_{0}^{\prime}>0$, as required to capture the behavior of polymer solutions.

Model predictions for constant values of the parameters $\varepsilon_{0}^{\prime}$ and $\varepsilon$ but with a different number of Kuhn segments $N$ are found to be identical, for both steady-state and transient quantities, for large values of $N(N \geq 10)$, because we have chosen to scale the UE viscosity with the modulus $G$ and the relaxation time $\lambda$, both of which do depend on $N$. For this reason, this comparison is not shown.

\section{Comparison with experimental data}

To test the steady-state, LVE, and transient predictions, we here compare against the rheological data (presented at $130{ }^{\circ} \mathrm{C}$ ) for UE provided by Huang et al. ${ }^{42}$ for one polystyrene polymer melt (PS-285k) and two polymer solutions (PS-285k/2k-72 and PS-285k/2k-44; volume fractions given in Table I). The

TABLE I. Parameters $Z, \lambda$, and $G$ employed in Figs. 5 and 6, while $\phi$ is the polymer volume fraction.

\begin{tabular}{lcrrc}
\hline \hline System & $\phi$ & $Z$ & $\lambda(\mathrm{s})$ & $G(\mathrm{MPa})$ \\
\hline PS-285k & 1.00 & 21.4 & 35000 & 1 \\
PS-285k-2k-72 & 0.72 & 15.4 & 2000 & 0.3 \\
PS-285k-2k-44 & 0.44 & 9.4 & 170 & 0.09 \\
\hline \hline
\end{tabular}




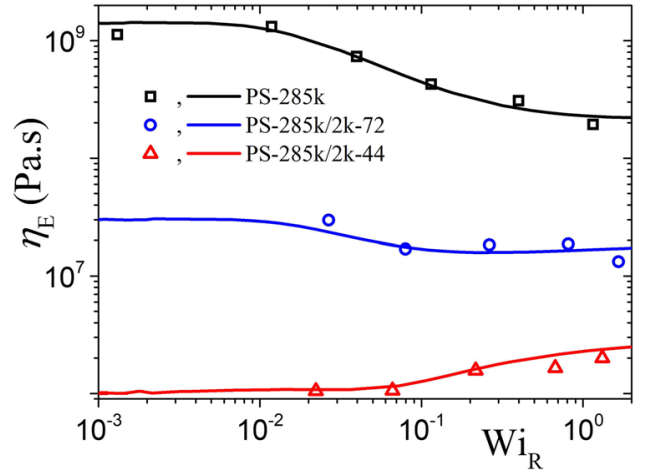

FIG. 5. Comparison of experimental data for the polymer melt (PS-285k) and two polymer solutions (PS-285k/2k-72 and PS-285k/2k-44) of Huang et al. $^{42}$ at $130{ }^{\circ} \mathrm{C}$ (symbols) with the tumbling-snake model predictions (lines) for the dimensionless, steady-state elongation viscosity as a function of $\mathrm{Wi}_{\mathrm{R}}=\dot{\boldsymbol{\epsilon}} \tau_{\mathrm{R}}$, where $\tau_{\mathrm{R}}=\tau_{d} / Z$ denotes the Rouse time.

values of the number of entanglements $Z$, the relaxation time $\lambda$, and the modulus $G$ are also included in Table I; the latter two were selected, for each sample, to obtain a good
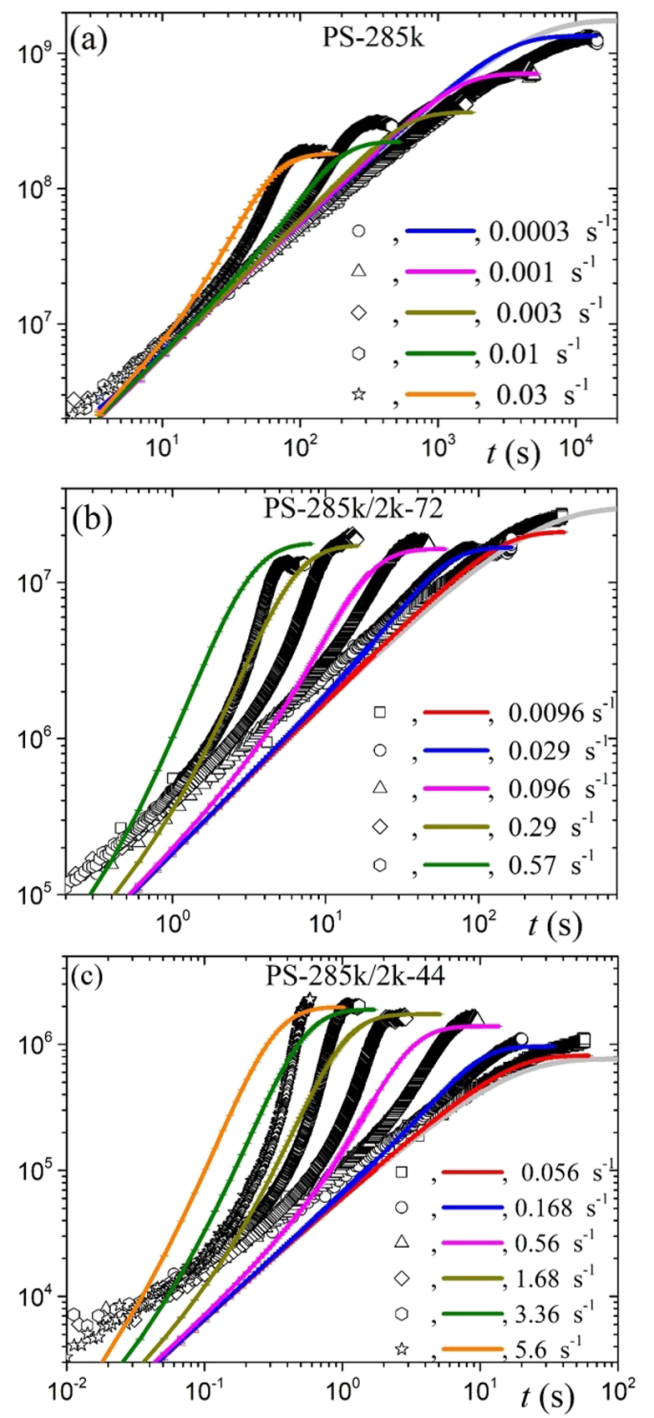

FIG. 6. Same as Fig. 5 but for the transient elongational viscosity as a function of time for (a) PS-285k, (b) PS-285k/2k-72, and (c) PS-285k/2k-44. The thick light gray lines in (a)-(c) depict the LVE envelopes according to Eq. (18). comparison with both the steady-state UE viscosity and the LVE envelope. The selected values for the moduli follow the expression $G(\phi)=G(1) \phi^{1+\alpha}$ using $\alpha=1$ as proposed by the same authors. ${ }^{42}$ We take $\varepsilon^{\prime}=0$ for the polymer melt, in accord with previous studies; ${ }^{14,20,23}$ for the polymer solutions, we consider $\varepsilon^{\prime}=0.1$ for PS-285k/2k-72 and $\varepsilon^{\prime}=0.5$ for PS$285 \mathrm{k} / 2 \mathrm{k}-44$. Finally, we obtained $\varepsilon_{0}$ by comparing the model results with the measured steady-state UE viscosity $(0.05$ for PS-285k, 0.2 for PS-285k/2k-72, and 0.9 for PS-285k/2k-44). The comparison with the steady-state UE viscosity is obviously very satisfactory (Fig. 5). The comparison with the transient UE viscosity for the polymer melt is also good, as shown in Fig. 6(a). However, as the polymer concentration decreases, the approach to the steady-state values is not matched quantitatively anymore, and it signals the regime of applicability of the tumbling-snake model developed for concentrated polymeric systems.

\section{CONCLUSIONS}

In this work, we provided the solution and discussed the features of the tumbling-snake model for concentrated solutions and entangled polymer melts subjected to both steady-state and transient UE. Following our recent work, we employed a variable link tension coefficient, given by $\varepsilon=\varepsilon_{0} S_{2}^{2}, 14,23$ which has amended several shortcomings of a constant link tension coefficient originally suggested by Bird et al. ${ }^{15,22}$ In particular, the UE viscosity does not approach a finite value as $t \rightarrow 0$ with the variable coefficient, because the system is isotropic at $t=0$, and $\varepsilon_{0}$ is proportional to the squared order parameter. We have demonstrated that the model is able to capture and interpret recent experimental evidence according to which the extensional viscosity of polymer solutions is seen to exhibit thinning below the inverse Rouse time and thickening above, whereas the extensional viscosity of polymer melts is monotonically decreasing for all strain rates. ${ }^{28,41-43}$ To this end, we found that the strength $\varepsilon_{0}$ of the link tension coefficient increases as the polymer concentration decreases (see Fig. 5). In other words, the friction tensor becomes more isotropic with decreasing concentration. While in the past the observed phenomena had been attributed to a flow-induced reduction of the monomeric friction coefficient, the tumbling-snake model keeps the monomeric friction coefficient constant but assumes a friction tensor that tends to become more isotropic with increasing $\varepsilon_{0}$ while attaining its maximum anisotropy at intermediate Wi. Using a friction tensor that is increasingly becoming isotropic at large strain rates as the polymer concentration decreases, the model is able to capture the observed behavior (see Fig. 5). However, the model fails to predict the experimentally observed behavior that, in some cases, as the polymer concentration decreases, the steady-state elongation viscosity first shear-thins and then reaches a minimum before it shear-thickens (e.g. Ref. 43). Also, the comparison against the time-dependent elongation viscosity is seen to become worse as the polymer concentration decreases (see Fig. 6). Such a disagreement may be due to the omission of mechanisms particularly important for elongational flows, such as chain stretch. 
Given the known capacity of the tumbling-snake model to provide a very adequate description of the shear rheological measurements of entangled polymer melts and concentrated polymer solutions, ${ }^{14,20,23}$ together with the results of the present study for the case of uniaxial elongation and those of Ref. 24 for planar elongation, we feel that this model bears the potential to improve our understanding regarding the rheological behavior of dense and entangled polymeric systems. It is now readily solved for arbitrary, including mixed homogeneous flow fields via $\mathrm{BD}$, and the meaning and choice of parameters had been discussed in detail for the extreme cases of academic flows. By properly introducing more refinements, such as contour length fluctuations, by considering a $\sigma$-dependent curvilinear segment diffusion along the polymer's contour (see, e.g., Refs. 6, 52, and 53 and references therein), uplifting the IAA by introducing a first-order derivative with respect to $s$ and a term proportional to the single-link distribution function, $2,25,54 \mathrm{CCR}$ by allowing for flow-induced relaxation times, ${ }^{10-12,55}$ flow-induced alignment of chain ends ${ }^{32,56}$ via a modification of the boundary conditions for the single-link distribution function, and chain stretch,,${ }^{8,57}$ could further improve the tumbling-snake's model capacity to favorably predict the rheological response of entangled polymer melts and concentrated polymer solutions. Especially, the latter is particularly important for strong elongation flows. All the possible refinements have not been done here to highlight the properties of the simple, unmodified model for future reference. These modifications to the tumbling-snake model should be made properly and selfconsistently by following the guidelines of non-equilibrium thermodynamics. ${ }^{58,59}$

\section{SUPPLEMENTARY MATERIAL}

See supplementary material for the methodology to obtain the real spherical harmonics expansion of the single-link distribution function and BD predictions for the elongational viscosity of the tumbling-snake model when a constant link tension coefficient is employed.

\section{ACKNOWLEDGMENTS}

We are grateful to Q. Huang for providing us with the rheological data. This work was co-funded by the Republic of Cyprus through the Research Promotion Foundation (Project No. KOYLTOYRA/BP-NE/0415/01), granted to P.S.S. through the "Cyprus Research Award-Young Researcher 2015" award.

\footnotetext{
${ }^{1}$ M. Doi and S. F. Edwards, J. Chem. Soc., Faraday Trans. 2 74, 1789 (1978).

${ }^{2}$ M. Doi and S. F. Edwards, The Theory of Polymer Dynamics (Clarendon, Oxford, 1986).

${ }^{3}$ P. G. de Gennes, J. Chem. Phys. 55, 572 (1971).

${ }^{4}$ H. Watanabe, Prog. Polym. Sci. 24, 1253 (1999).

${ }^{5}$ T. C. B. McLeish, Adv. Phys. 51, 1379 (2002).

${ }^{6}$ P. S. Stephanou and V. G. Mavrantzas, J. Non-Newtonian Fluid Mech. 200, 111 (2013).

${ }^{7}$ P. S. Stephanou and V. G. Mavrantzas, J. Chem. Phys. 140, 214903 (2014).

${ }^{8}$ G. Marrucci and N. Grizzuti, Gazz. Chim. Ital. 118, 179 (1988).

${ }^{9}$ G. Ianniruberto and G. Marrucci, J. Rheol. 45, 1305 (2001).

${ }^{10}$ P. S. Stephanou, I. C. Tsimouri, and V. G. Mavrantzas, Macromolecules 49, 3161 (2016).
}

${ }^{11}$ G. Marrucci, J. Non-Newtonian Fluid Mech. 62, 279 (1996).

${ }^{12}$ G. Ianniruberto and G. Marrucci, J. Non-Newtonian Fluid Mech. 65, 241 (1996).

${ }^{13}$ G. Ianniruberto and G. Marrucci, Philos. Trans. R. Soc., A 361, 677 (2003).

${ }^{14}$ P. S. Stephanou, T. Schweizer, and M. Kröger, J. Chem. Phys. 146, 161101 (2017).

${ }^{15}$ R. B. Bird, R. C. Armstrong, and O. Hassager, Dynamics of Polymeric Liquids: Volume 2, Kinetic Theory (John Wiley \& Sons, New York, 1987).

${ }^{16}$ J. D. Schieber and M. Andreev, Annu. Rev. Chem. Biomol. Eng. 5, 367 (2014).

${ }^{17}$ Y. Masubuchi, Annu. Rev. Chem. Biomol. Eng. 5, 11 (2014).

${ }^{18}$ C. F. Curtiss and R. B. Bird, J. Chem. Phys. 74, 2016 (1981).

${ }^{19}$ C. F. Curtiss and R. B. Bird, J. Chem. Phys. 74, 2026 (1981).

${ }^{20}$ P. S. Stephanou and M. Kröger, J. Chem. Phys. 144, 124905 (2016).

${ }^{21}$ R. B. Bird, H. H. Saab, and C. F. Curtiss, J. Chem. Phys. 77, 4747 (1982).

${ }^{22}$ R. B. Bird, H. H. Saab, and C. F. Curtiss, J. Phys. Chem. 86, 1102 (1982).

${ }^{23}$ P. S. Stephanou and M. Kröger, J. Chem. Phys. 147, 174903 (2017).

${ }^{24}$ P. S. Stephanou and M. Kröger, Polymers 10, 329 (2018).

${ }^{25} \mathrm{H}$. C. Öttinger, Stochastic Processes in Polymeric Fluids: Tools and Examples for Developing Simulation Algorithms (Springer, Berlin, 1996).

${ }^{26}$ T. Schweizer, J. Hostettler, and F. Mettler, Rheol. Acta 47, 943 (2008).

${ }^{27}$ D. Auhl, J. Ramirez, A. E. Likhtman, P. Chambon, and C. Fernyhough, J. Rheol. 52, 801 (2008).

${ }^{28}$ S. Costanzo, Q. Huang, G. Ianniruberto, G. Marrucci, O. Hassager, and D. Vlassopoulos, Macromolecules 49, 3925-3935 (2016).

${ }^{29}$ M. H. N. Sefiddashti, B. J. Edwards, and B. Khomami, J. Rheol. 59, 119 (2015)

${ }^{30}$ M. H. N. Sefiddashti, B. J. Edwards, and B. Khomami, J. Rheol. 60, 1227 (2016).

${ }^{31}$ J. M. Kim and C. Baig, Sci. Rep. 6, 19127 (2016).

${ }^{32}$ M. Kröger, Models for Polymeric and Anisotropic Liquids (Springer, New York, 2005), Vol. 675.

${ }^{33}$ R. G. Larson, The Structure and Rheology of Complex Fluids (Oxford University Press, New York, 1999).

${ }^{34}$ C. Luap, C. Müller, T. Schweizer, and D. C. Venerus, Rheol. Acta 45, 83 (2005).

${ }^{35}$ M. Kröger and S. Hess, Macromol. Symp. 81, 83 (1994).

${ }^{36}$ D. Malkus and B. Bernstein, J. Non-Newtonian Fluid Mech. 16, 77-116 (1984).

${ }^{37}$ B. Bernstein, D. S. Malkus, and E. T. Olsen, Int. J. Numer. Methods Fluids 5, 43 (1985).

${ }^{38}$ R. Wesson, T. Papanastasiou, and J. Wilkes, J. Non-Newtonian Fluid Mech. 32, 157 (1989).

${ }^{39}$ P. K. Currie, J. Non-Newtonian Fluid Mech. 11, 53-68 (1982).

${ }^{40}$ A. Bach, K. Almdal, H. K. Rasmussen, and O. Hassager, Macromolecules 36, 5174 (2003).

${ }^{41}$ Q. Huang, N. J. Alvarez, Y. Matsumiya, H. K. Rasmussen, H. Watanabe, K. Almdal, and O. Hassager, ACS Macro Lett. 2, 741 (2013).

${ }^{42}$ Q. Huang, O. Mednova, H. K. Rasmussen, N. J. Alvarez, A. L. Skov, K. Almdal, and O. Hassager, Macromolecules 46, 5026 (2013).

${ }^{43}$ Q. Huang, L. Hengeller, N. J. Alvarez, and O. Hassager, Macromolecules 48, 4158 (2015).

${ }^{44}$ P. K. Bhattacharjee, J. P. Oberhauser, G. H. McKinley, L. G. Leal, and T. Sridhar, Macromolecules 35, 10131 (2002).

${ }^{45}$ T. Yaoita, T. Isaki, Y. Masubushi, H. Watanabe, G. Ianniruberto, A. Brasiello, and G. Marrucci, Macromolecules 45, 2773 (2012).

${ }^{46} \mathrm{G}$. Ianniruberto, A. Brasiello, and G. Marrucci, Macromolecules 45, 8058 (2012).

${ }^{47}$ P. S. Desai and R. G. Larson, J. Rheol. 58, 255 (2014).

${ }^{48}$ G. Ianniruberto, Macromolecules 48, 6306 (2015).

${ }^{49}$ H. Giesekus, J. Non-Newtonian Fluid Mech. 11, 69 (1982).

${ }^{50}$ R. B. Bird and J. R. DeAguiar, J. Non-Newtonian Fluid Mech. 13, 149 (1983).

${ }^{51}$ R. B. Bird, H. R. Warner, and D. C. Evans, Adv. Polym. Sci. 8, 1 (1971).

${ }^{52}$ P. S. Stephanou, C. Baig, and V. G. Mavrantzas, Macromol. Theory Simul. 20, 752 (2011).

${ }^{53}$ P. S. Stephanou, C. Baig, and V. G. Mavrantzas, Soft Matter 7, 380 (2011).

${ }^{54}$ M. Doi, J. Polym. Sci. 18, 2055 (1980).

${ }^{55}$ H. C. Öttinger, J. Non-Newtonian Fluid Mech. 89, 165 (2000).

${ }^{56}$ M. Kröger and S. Hess, Phys. A 195, 336 (1993).

${ }^{57}$ J. Fang, M. Kröger, and H. C. Öttinger, J. Rheol. 44, 1293 (2000).

${ }^{58}$ H. C. Öttinger, J. Rheol. 43, 1461 (1999).

${ }^{59}$ H. C. Öttinger, Beyond Equilibrium Thermodynamics (Wiley-Interscience, Hoboken, 2005) 